\title{
A Cooperative Car-Following/Emergency Braking System With Prediction-Based Pedestrian Avoidance Capabilities
}

\author{
Carlos Flores ${ }^{(}{ }^{\circ}$, Pierre Merdrignac ${ }^{\circledR}$, Member, IEEE, Raoul de Charette, \\ Francisco Navas, Vicente Milanés, and Fawzi Nashashibi
}

\begin{abstract}
Urban environments are among the most challenging scenarios for car-following systems, since pedestrians may interfere with the platoon unexpectedly. To address this problem, this paper proposes a cooperative system using vehicle-to-vehicle and vehicle-to-pedestrian communication links. A fractionalorder control-based cooperative adaptive cruise control benefits of communication for tighter inter-vehicle distances, while pedestrian communication is fused with LiDAR sensing to allow the detection of occluded pedestrians. The prediction of the pedestrians' trajectories is used to perform a speed reduction or an emergency braking that interrupts the car-following yif necessary. Whenever a platoon decoupling occurs, a gap-closing maneuver is executed so that the ego-vehicle rejoins the platoon in a string stable way. The complete system was tested on experimental platforms at inria facilities, providing encouraging results and demonstrating the correct performance of the integrated systems.
\end{abstract}

Index Terms-Cooperative systems, fractional calculus, intelligent transportation system (ITS), sensor fusion, collision avoidance system.

\section{INTRODUCTION}

C OOPERATIVE systems based on vehicular communications are getting more attention because of their potential benefits to increase traffic safety and flow. Vehicles can take advantage of a wireless link by receiving additional information from either other vehicles-i.e. vehicle-to-vehicle (V2V)the infrastructure (V2I) or even from pedestrians (V2P), generally referred as V2X communication [1]. In recent years, several V2X-based techniques have been developed such as the evolution of the Adaptive Cruise Control (ACC), the Cooperative-ACC (CACC) [2], or the intelligent vehicle speed control to improve traffic congestion based on V2I

Manuscript received July 11, 2017; revised January 26, 2018 and March 26, 2018; accepted May 16, 2018. This work was supported by the French National Research Agency through the VALET Project under Grant ANR-15-CE22-0013. The Associate Editor for this paper was L. Li (Corresponding author: Carlos Flores.)

C. Flores, R. de Charette, F. Navas, and F. Nashashibi are with the Robotics and Intelligent Transportation Systems Team, inria, 75012 Paris, France (e-mail: carlos.flores-pino@inria.fr; raoul.de-charette@inria.fr; francisco.navas@inria.fr; fawzi.nashashibi@inria.fr).

P. Merdrignac is with the VEDECOM Institute, 78000 Versailles, France (e-mail: pierre.merdrignac@vedecom.fr).

V. Milanés is with the Research Department, Renault SAS, 78280 Guyancourt, France (e-mail: vicente.milanes@renault.com).

Color versions of one or more of the figures in this paper are available online at http://ieeexplore.ieee.org.

Digital Object Identifier 10.1109/TITS.2018.2841644

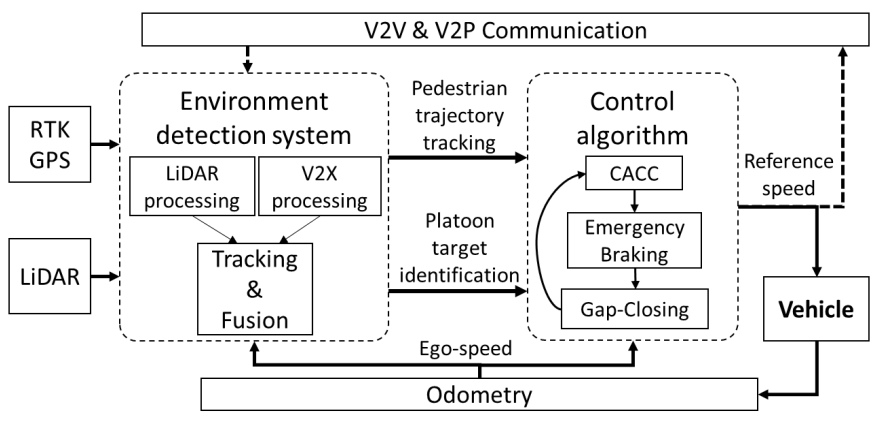

(a)

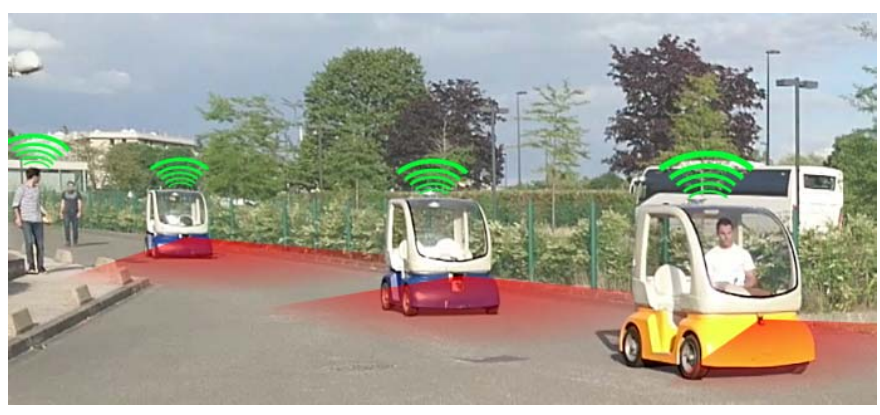

(b)

Fig. 1. Overview of the proposed architecture and its experimental validation. (a) General outlook of the system. (b)Picture of the experiment.

communication [3]. CACC systems capability to enhance traffic flow has been demonstrated for highway driving [4]. However, their development on urban environments remains as an unsolved challenge due to multiple interactions with non-motor vehicles, constant speed variation and the requirement of more demanding algorithms. As a matter of fact, almost half of all deaths on the world's roads are among the most vulnerable actors, specially pedestrians [5]. Consequently, the system has to be able not only to properly follow the preceding vehicle in a stable way but also to deal with unexpected pedestrians that can interact in the platoon trajectory, specially for urban environments.

A state-of-the-art review shows an extensive work on active pedestrian protection systems for urban environments based on vision [6], laser [7], ultrasound systems [8] or multi-sensor fusion [9]. However, fully reliable active pedestrian protection 
systems [10] still represent a major challenge mostly because the field of view in urban scenarios is cluttered up by other road users and buildings. The most common strategy to leverage occlusion in perception system is the inference of objects geometry as in [11] and [12]. Such strategy is limited to partial occlusion of non-deformable objects. One of the hot topics on cooperative systems is the benefits that V2P communications can bring to pedestrian protection thanks to the high market penetration of wireless-equipped personal devices-i.e. tablets or smartphones. Profiting from these links, pedestrian position can be estimated even when occluded and its future path predicted to prevent potential collisions. There are just a few works on the literature related to this topic. In [13], a vehicle-to-pedestrian warning system was implemented informing the pedestrian about potential dangerous situations. Zhenyu et al. [14] presents a warning system for both pedestrian and drivers using V2P communications but an active $\mathrm{V} 2 \mathrm{P}$ pedestrian protection system has not been yet investigated.

Among the most recent advances in low-speed carfollowing systems, one can find in [15] a safety distance policy for ACC-equipped vehicles. Such spacing policy was later implemented on [16], where two different control laws were compared and tested on an experimental vehicle. Moon et al. [17] developed a full-range ACC with collision avoidance capabilities that was also validated on a real vehicle. However, all these papers addressed the problem by only using range sensors without exploiting the benefits of adding V2X capabilities. Furthermore, platoon splitting/rejoining or the interaction with other transport agents have not been further investigated.

This paper presents a novel low-speed V2X CACC system with emergency braking and formation rejoining capabilities in case of stopping, (see Fig. 1a). The main functionalities of the proposed system are as follows:

- V2V communication to achieve tighter inter-vehicle distances in the platoon and V2P communication for predicting potential pedestrian interactions.

- System for environment perception, particularly obstacles detection and estimation of their dynamics (position and velocity) from fusion of LiDAR and V2P data, identifying the front target vehicle in the platoon and tracking pedestrians with which a collision may occur.

- Control system based on a state machine able to ensure gap-regulation stability and robustness employing fractional-order calculus. Such systems also handles pedestrian interaction through an emergency braking state and a further platoon rejoining through a gap closing maneuver.

The whole system has been tested on real experimental platforms at INRIA test tracks, showing a promising performance to introduce CACC systems at low-speed with additional safety considerations for pedestrians.

The rest of the paper is structured as follows. Section 2 presents the experimental platform that is used for the system validation and the on-board vehicle equipment. Section 3 describes the environment detection algorithm. The control algorithm is introduced in Section 4. Experimental results using the INRIA platforms are included in Section 5. Finally, some concluding remarks are given in Section 6.

\section{EXPERIMENTAL SETUP}

Three fully automated cars called $C y c a b$ are employed for the experiments on INRIA test track (Fig. 1b). Cycab motion is powered by four in-wheel electric motors. Their speed is regulated by a low-level controller in function of a target speed and the vehicle current speed.

The mentioned platforms count with an odometer, an Ashtech Z-Xtrem RTK GPS, an Ibeo LiDAR, and a Yawarra Alix-3 communication box. Along with the odometer, GPS measurements are filtered with an Extended Kalman Filter (EKF) [18] which improves localization robustness significantly in urban conditions.

A front Ibeo LiDAR provides a $110^{\circ}$ Field Of View with an angular resolution of $0.125^{\circ}$. Even though the LiDAR provides several layers, only the most parallel to the ground ${ }^{1}$ is used to remain close to industrial setup. Communication link is established using IEEE $802.11 \mathrm{~g}$ standard because the available boxes do not account with $802.11 \mathrm{p}$ standard $^{2}$ and platoon leader reference speed is broadcast at a minimum rate of $100 \mathrm{~Hz}$. Besides, a pedestrian carries a Samsung tablet for broadcasting its position and velocity similarly to [13].

Processing of the sensors data as well as communication with the low level control are implemented in $\mathrm{C}++$ using the RTMaps prototyping software.

\section{EnVironment Detection System}

To leverage the common problem of scene occlusion, the approach combines LiDAR and Vehicle-toPedestrian (V2P) communication to sense the scene and track objects. The use of a LiDAR over camera is motivated by its ability to sense the geometry, allowing better estimation of the dynamics of the objects at a small processing cost. The single threaded detection algorithm runs under $2 m s$ which allows frequent update to the control algorithm. Both LiDAR (Sec. III-A) and V2P (Sec. III-B) processing runs in parallel and the resulting objects are merged and tracked through time with a Multiple Hypothesis Tracking (MHT) filter (Sec. III-C). Lastly, to decide which detected objects should be classified as an obstacle and activate the emergency braking, both platoon and critical stopping strategies are handled by a decision scheme described in Sec. III-D.

\section{A. LiDAR Processing}

Each LiDAR frame forms 2D layer point cloud, capturing a slice of the scene geometry parallel to the ground. A split-andmerge algorithm-i.e. Ramer-Douglas-Peucker algorithm [19] (RDP)-is used to segment the point cloud. This algorithm is popular in the literature [19]-[22] and was shown to exhibit similar performance than RANSAC, Hough Transform and Expectation Maximization while being at least 16 times

\footnotetext{
${ }^{1}$ This is determined experimentally with a cone-like object (with height similar to the LiDAR height) imaged at different distances.

${ }^{2}$ Replacement of $11 \mathrm{~g}$ with $11 \mathrm{p}$ should be straightforward, when available.
} 


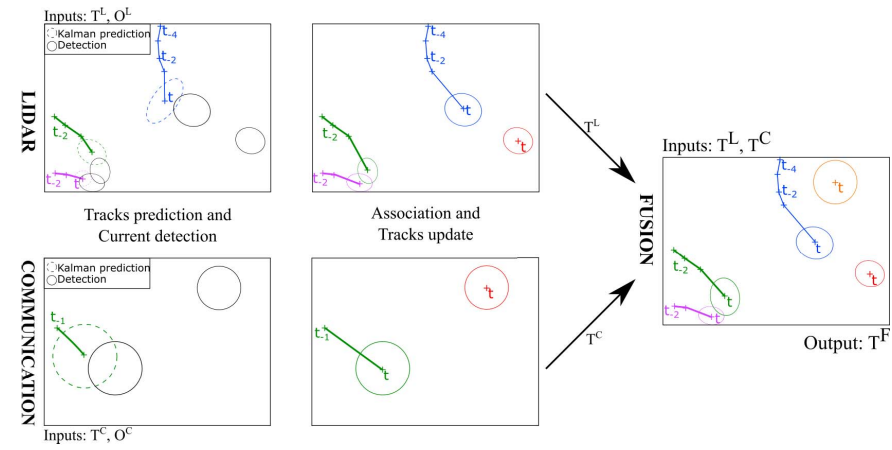

Fig. 2. Illustration of the tracking and fusion strategy. Refer to text in section III-C for details.

faster [21]. The proposed implementation of the RDP also benefits from the LiDAR polar sorting behavior, which makes it an $O(n \log n)$ complexity [20]. This contributes to the fast reactive control of the whole system. The segments obtained are grouped using the implicit polar property that object are imaged as a set of adjacent segments. Following this observation, each object is built through the parsing of the angle-sorted set of segments with non-adjacent pair of segments initiating a new object. As highlighted in [11] the adjacent property can be found false in the case of partial occlusion or objects with holes but for the current platoon application this limitation was found acceptable. The classification used is very simple but efficient and stable enough when used in conjunction with the tracking strategy that temporally refines classification as in [23].

The LiDAR processing described is capable of detecting all objects in the sensor field of view. This set of detected objects is denoted as $\left\{O_{1}^{L}, \ldots, O_{n}^{L}\right\}$ (where $L$ stands for LiDAR). In the experiments, detection and classification of cars/pedestrian are working robustly in most of the encountered scenarios.

\section{B. V2P Communication}

V2P was recently introduced to improve pedestrian safety by relying on either centralized [24] or distributed communication protocols [25]. Similar to our work [26], in this work pedestrians carry a communication system sending Pedestrianto-Vehicle (P2V) messages with geographic location, speed, walking direction and user type. As for Cooperative Awareness Messages (CAM) [27], it allows tracking a communicating user even when not detected with LiDAR. Upon the reception of P2V message the relative position of the emitter is calculated using the World Geodetic System (WGS84) [28].

The main limitation of this approach lies in the low precision of the GPS hand-held device. Our previous evaluation [13] showed that $95 \%$ of GPS position has an error below $10 \mathrm{~m}$ and an exhaustive study [29] stated that GPS from the best smartphone at that time had of $4.68 \mathrm{~m}$ and $6.83 \mathrm{~m}$ longitudinal and lateral variance, respectively. Although the precision is too low for the emergency braking system to rely on, the large communication range allows detecting the risk of a car-pedestrian collision if both are tracked [13].
In this experiment the freshness of data transmitted using V2P communication is evaluated to ensure an accurate and up-to-date pedestrian localization. Results of the experiment show that the average end-to-end V2P latency ${ }^{3}$ is $36.5 \mathrm{~ms}$ with a standard deviation of $1.6 \mathrm{~ms}$. Noteworthy, $97 \%$ of the packets are received within $40 \mathrm{~ms}$, which is sufficient for the targeted system.

Given that V2P communication is asynchronous, the P2V messages received during an interval of time are stored as set of detected objects $\left\{O_{1}^{C}, \ldots, O_{n}^{C}\right\}$ (where $C$ stands for Communication) for further fusion with LiDAR information.

\section{Tracking and Fusion}

To get a complete environment sensing, the system needs to estimate the dynamics of these objects (trajectory, speed, acceleration) through tracking of the detected objects. This is a prerequisite to any decision making process for autonomous vehicles as it should behave differently towards objects with different dynamics. The problem arising from tracking objects detected with LiDAR and communication processing in parallel, is that the sets of detected objects might be either redundant or complementary thus leading to conflicts or duplicates in the detection process. For example, a pedestrian might be detected with the LiDAR (i.e. an $O^{L}$ instance) and as a communicating pedestrian (i.e. an $O^{C}$ instance), leading to two distinct objects if merged naively. To leverage that problem, LiDAR and communication objects are tracked separately and redundant tracks are then fused. The literature on objects tracking is vast and includes a large variety of algorithms [30], [31] like geometrical tracking, particle filtering, Kalman filter, and Gaussian Mixture Models to name a few. A Multiple Hypothesis Kalman Filter [32] is employed in this work due to its extended usage.

The tracking and fusion strategy is described in Algo. 1. Each set of object $O^{L}$ and $O^{C}$ is filtered through time with a multi-track Kalman Filter and outputs sets of tracks, respectively $T^{L}$ and $T^{C}$. The filter takes advantage of the transition model (often denoted $F_{t}$ ) and the control matrix $\left(B_{t}\right)$ that can be easily computed from the derivative with respect to time. The control vector $\left(u_{t}\right)$ of the observer - i.e. ego-vehicle used to predict the a priori state estimate, is:

$$
u_{t}=\left(\begin{array}{l}
-v_{t} * \cos \theta \\
-v_{t} * \sin \theta
\end{array}\right)
$$

with $v_{t}$ and $\theta$ the velocity and relative orientation of the ego-vehicle respectively.

For each LiDAR time step, the a priori state is matched against all $O^{L}$ objects (observations); and the object minimizing the Mahalanobis distance [33] is associated with the track and serves as posteriori state estimate of the Kalman Filter. As usual, only object-track pairs with Mahalanobis distance below a threshold $\sigma_{d}$ are associated; and non-associated objects initialize new tracks-i.e. new Kalman Filters-. Since the position error is fairly Gaussian, the value of $\sigma_{d}$ can be defined using the quartile function of the chi-squared

\footnotetext{
$3_{i}$ i.e. Time difference between reception and emission using GPS time.
} 


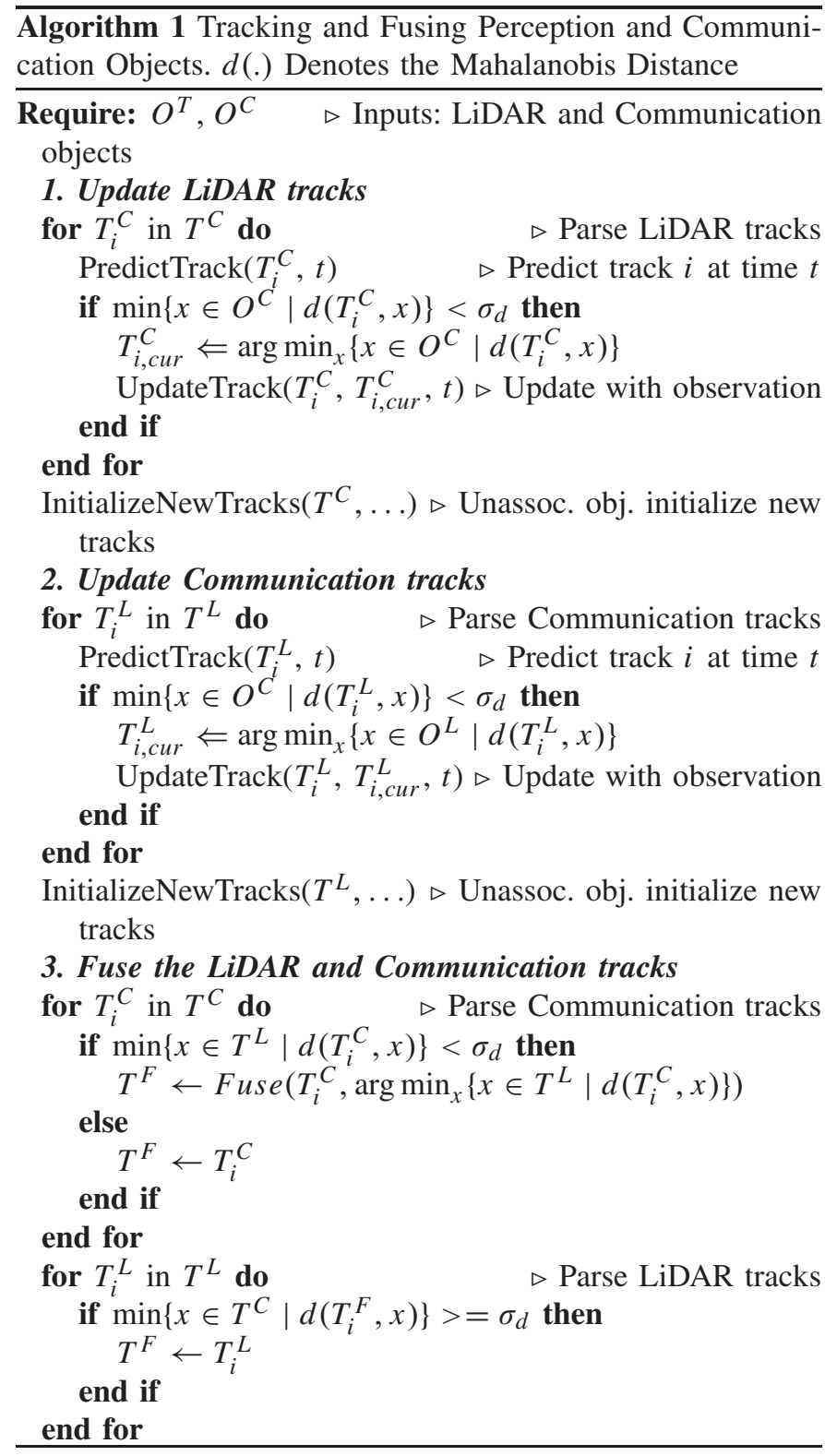

distribution with two degrees of freedom ${ }^{4}$ for a probability of $90 \%$ that is:

$$
\sigma_{d}=\mathcal{X}_{1-0.9,2}^{2}=4.605
$$

For each V2P communication time step, the a priori state is matched against $O^{C}$ objects. The object-track association is straightforward since communication broadcasts identifiers. However, because messages are asynchronous, the state of a track is propagated only with the prediction if no new P2V was received in the time interval.

Fig. 2 illustrates the steps of the tracking and fusion, from left to right: detection of LiDAR/Communication objects and prediction of existing tracks, update of the LiDAR/Communication tracks, and fusion of both. The fusion avoids duplicated pedestrians due to sensors redundancy. In practice, the communication $T^{C}$ and LiDAR tracks $T^{L}$ are fused into a final set denoted $T^{F}$ if tracks have a Mahalanobis

\footnotetext{
${ }^{4}$ Recall obstacles lie on a $2 \mathrm{D}$ plane due to the use of single layer, hence 2 degrees of freedom.
}

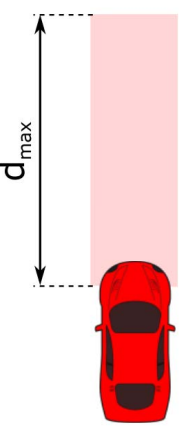

(a)

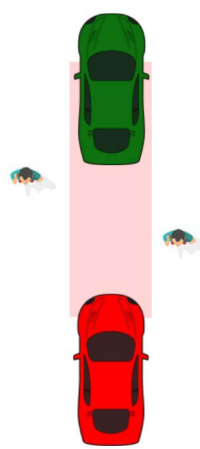

(b)

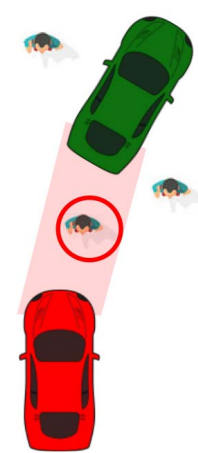

(c)
Fig. 3. Our platoon strategy. The system starts without target (3a). Target identification is achieved through intersection with the trajectory corridor (3b). The ego-target platoon corridor serves as detection for obstacles, circled in red (3c). (a) No target. (b) Target identification. (c) Target following.

distance below the same threshold $\sigma_{d}$. Inherently, tracking requires at least two observations which occurs at worse after $80 \mathrm{~ms}$ or $160 \mathrm{~ms}$ for communicating or lidar obstacles, allowing our system to react faster than a human.

\section{Platoon Strategy}

Fig. 3 shows the three platoon states considered in the experiment: a) No target, b) Target identification, c) Target following. The no target state is self-explanatory (Fig. 3a). The second state (Fig. 3b) is the identification of the preceding vehicle among the fused tracks $\left(T^{F}\right)$; that is the closest vehicle intersecting the trajectory corridor. Because experiments were conducted in straight line the trajectory corridor is similar to the projection of vehicle's width along its longitudinal axis. As the target is identified, it is tracked and the trajectory corridor is now computed as the polygon formed by the ego vehicle and the target (Fig. 3c). Any tracks intersecting the trajectory corridor is considered an obstacle.

Each of the platoon vehicles has the presented detection system embedded. Except for the leader vehicle, the platoon strategy scheme identifies each target vehicle and in-between pedestrians to ensure their safety. On each vehicle, the control algorithm takes as inputs the relative coordinates of the target, the reference velocity of the preceding vehicle in the platoon (V2V), as well as the list of identified obstacles.

\section{Control Algorithm}

The control algorithm proposed focuses on the longitudinal vehicle dynamics and should handle platoon splitting/joining as well as pedestrian interaction. A state machine was designed to allow proper integration of the different control strategies (see Fig. 4). The lateral dynamic was handled by using a joystick with drive-by-wire system. If lateral automation is desired, further implementation of a path-following controller can be considered [34].

The leader vehicle executes a cruise control following a reference speed profile with several speed changes, emulating urban driving. Pedestrian interaction can be predicted through 


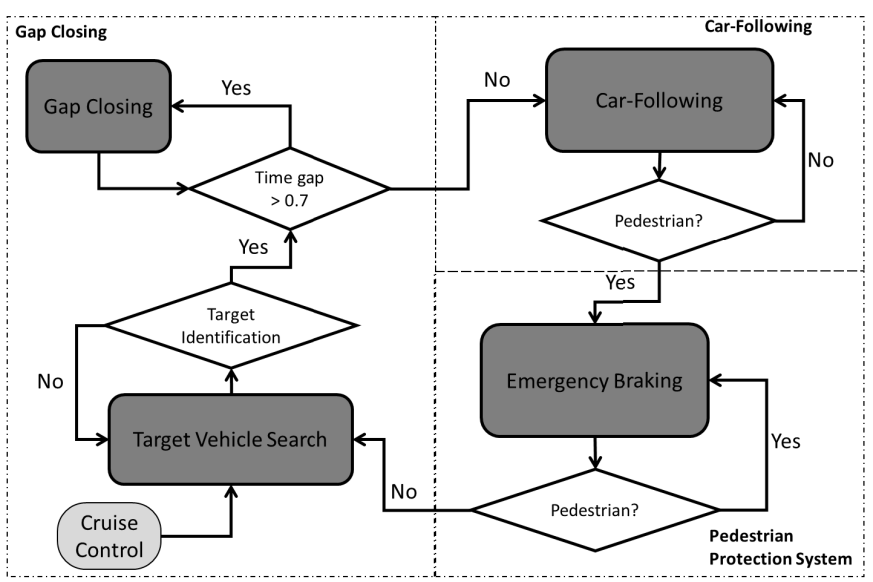

Fig. 4. State machine which describes the control algorithm.

the environment detection system. If so occurs, the speed profile is interrupted and the speed reduced, avoiding future collision. On the other hand, if the pedestrian is predicted to interfere with any of the other vehicles in the platoon, an emergency braking response has to be carried out since inter-vehicular distances are tight.

For the sake of clarity, the starting point in the state machine is the cruise controller. Then, three main states are identified: CACC car-following, emergency pedestrian protection system and gap closing. The explanation assumes as initialization point that all vehicles are connected in a car-following. Such proposed algorithm is presented in the remaining of this section.

\section{A. Car-Following Control (CACC)}

The initial state is the car-following control. Each vehicle will track its preceding one regulating the distance gap with respect to the desired inter-distance provided by a spacing policy. Constant time gap strategy [35] is employed in this work, which proposes a reference spacing defined as:

$$
d_{r e f}(t)=d_{s t d}+h_{d} \cdot v(t)
$$

having $d_{s t d}$ as the fixed standstill distance and a variable term composed by the vehicle longitudinal speed $v(t)$ multiplied by a constant time gap $h_{d}$.

Different approaches for the CACC control algorithms have been proposed in the literature, such as model predictive control (MPC) [36], sliding-mode control [37], $\mathcal{H}_{\text {inf }}$ controller-based approaches [38], Lyapunov-based cooperative braking control [39] and feedforward control [40]. The latter is selected for this work due to its simplicity and the good results that it has provided in the literature [2], [38], [41]; making it ideal for embedded systems implementations. This technique allows to use filtered information received from the preceding vehicle together with the feedback controller output. Fig. 5 shows the control structure, including the spacing policy defined in Eq. 3 represented as $H(s)=h_{d} \cdot s+1$ in the feedback loop. The block $D(s)$ stands for the transport delay due to the V2V communication link, which affects directly the reaction speed that the ego-vehicle has towards leader speed

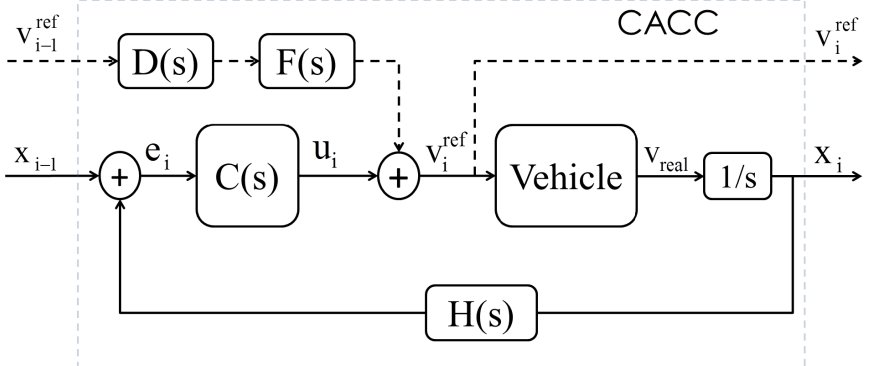

Fig. 5. Block diagram which illustrates the CACC control approach of the "i" vehicle.

changes. The feedforward filter is selected as $F(s)=H(s)^{-1}$ to guarantee theoretical string stability if no delay is assumedi.e. $D(s)=e^{-\theta s}=1[41]$.

1) Controller Design: The vehicle dynamics are modelled by a second order function of the form:

$$
G p(s)=\frac{v_{\text {real }}(s)}{v_{\text {ref }}(s)}=\frac{1}{1+0.2551 s+0.1514 s^{2}}
$$

which describes the dynamic response of the low-level control in charge of following the desired speed $v_{\text {ref }}$. Among the state-of-the-art approaches of car-following gap regulation control [2], proportional derivative (PD) controllers work well with feedforward structures due to their damping and stabilizing capabilities. This work proposes a FOPD to keep PD performance but also increase design flexibility profiting from fractional order derivation. FOPD [42] can be mathematically expressed in the Laplace domain as:

$$
C(s)=\frac{U(s)}{E_{d}(s)}=K p+K d \cdot s^{\alpha} ;
$$

Since there are three design parameters in the control law, three requirements can be fulfilled. In this work, it is proposed to employ a design procedure that ensures a system response robust towards loop gain variations [43] for CACC carfollowing. In other words, the controller will maintain the desired phase margin even if disturbances slightly modify the plant DC gain. Consequently, the design requirements are stated as follows:

1) Systems phase margin $\phi_{m} \approx \frac{\pi}{2}$ :

$$
\arg \left(G p\left(j \omega_{g c}\right) \cdot C\left(j \omega_{g c}\right)\right)=-\pi+\phi_{m}
$$

2) Increase the system bandwidth. In other words, the gaincross frequency of the open loop response should be increased to get faster responses.

3) Robustness against loop gain variations, or flat phase in $\omega=\omega_{g c}$ :

$$
\left(\frac{d\left(\arg \left(G p\left(j \omega_{g c}\right) \cdot C\left(j \omega_{g c}\right)\right)\right)}{d \omega}\right)=0
$$

After applying the algorithm $f$ solve from Matlab to solve the resulting multi-objective optimization problem for non-linear functions, the best configuration of the controller parameters results: $K p=2.66, K d=0.79$ and $\alpha=0.93$. The bode plot presented in Fig. 6a shows the plants' (blue), controllers' (green) and the open loop (red) responses. 


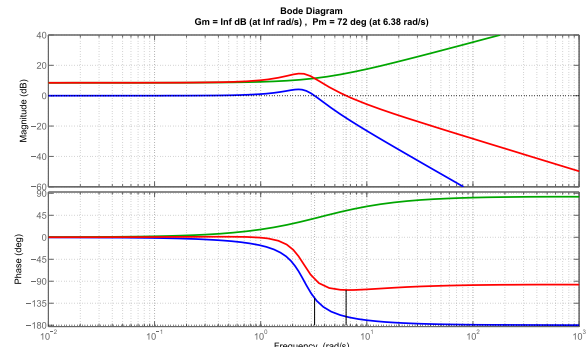

(a)

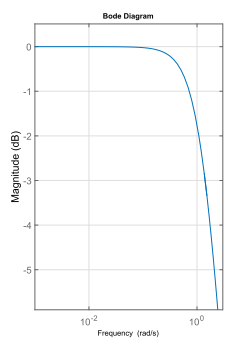

(b)
Fig. 6. Frequency study of the open and closed loop response of the system. (a) Bode analysis of the loop. (b) String stability transfer function.

As it can be distinguished in Fig. 6a, the systems' bandwidth is increased and the flat phase around the gaincross frequency is achieved, guaranteeing that the system will keep the same stability and dynamic behavior in case of variations on the loop gain. String stable performance is obtained as one can see in Fig. 6b that $\left\|\frac{X_{i}(s)}{X_{i-1}(s)}\right\|_{\infty} \leq 1$.

\section{B. Emergency Pedestrian Protection System}

In case a pedestrian crosses between the string members, the control algorithm must to react safely at any time and break the string formation to avoid a collision. In the literature, a vision based approach has been done for automatic stopping in case of pedestrian [44]. Furthermore, a very probable scenario is that the pedestrian is detected by the environment detection system (Sec. III) only at the moment he/she enters the platoon corridor, which may be caused by a visual occlusion with another car, tree, corner, etc. For these cases, the control algorithm must interrupt the car-following maneuver and switch to the emergency pedestrian protection system.

Fig. 7 illustrates the working system, where two string members are driving at the cruise speed $v_{0}$ and a pedestrian enters the platoon corridor. At the detection moment, the egovehicle starts the braking maneuver taking into consideration the distance to the pedestrian $d_{\text {detection }}$ (or the closest one in case of more than one detection).

Right at the crossing moment, an estimation of the vehicle deceleration ramp is carried out as:

$$
a_{\text {ref }}=\frac{v_{o}^{2}}{2 \cdot\left(d_{\text {detection }}-d_{\text {safety }}\right)}<a_{\text {max }}
$$

where $v_{0}$ is the string cruise speed. The term $a_{\text {max }}$ stands for the maximum deceleration reachable by the vehicle. With some manipulation, Eq. (8) allows to determine also the minimal $d_{\text {detection }}$ for a given $v_{0}$ for which the emergency braking maneuver is feasible. The vehicle should decelerate with the required rate to stop at a safe distance $d_{\text {safety }}$ from the pedestrian, avoiding any collision. Taking this estimation as reference, the desired speed is outputted in real time in function of the distance to the pedestrian received from the environment detection system:

$$
v_{\text {ref }}(t)=\sqrt{v_{0}^{2}-2 \cdot a_{\text {ref }} \cdot\left(d_{\text {detection }}-d_{\text {safety }}-d_{2 p e d}(t)\right)}
$$

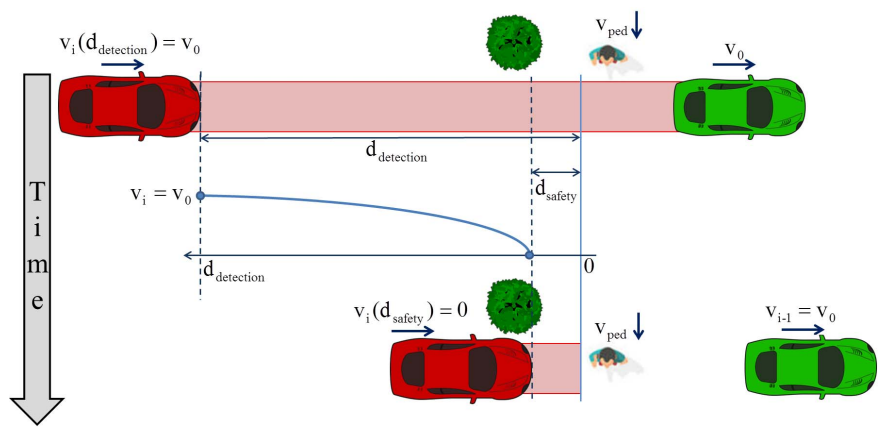

Fig. 7. Desired behavior in case that a pedestrian crosses the string corridor and an emergency braking is required.

To ensure a safe and accurate execution of the stopping maneuver, a speed controller modifies the reference speed sent to the low level control in function of the desired speed in 9 and the measured ego-speed. The selected controller is a PD for its damping properties and stabilizing behavior. The mentioned controller is described by the control law showed in the Eq. 10.

$$
u(t)=\left(K p+K d \cdot \frac{d}{d t}\right)\left(v_{r e f}(t)-v(t)\right)
$$

\section{Gap Closing Algorithm}

Once the pedestrian is no longer inside the platoon corridor, the ego-vehicle can safely rejoin the platoon formation. However, in practice, the difference between the reference distance $d_{\text {ref }}$ and the relative spacing $d(t)$ measured by the LiDAR may result really large after the stopping maneuver. This would lead to saturation and oscillations in the controller [45]. To avoid this scenario, preserve stability and ensure passengers' comfort; a gap closing maneuver is required.

The applied condition to ensure no saturation and oscillation when performing the gap regulation, is that the controller input (spacing error $e_{d}$ ) is zero just after finishing emergency braking state. This is attained when $h_{d}$ and the measured time gap $h_{d, \text { meas }}$ are equal. The latter is calculated as:

$$
h_{d, \text { meas }}(t)=\frac{d(t)-d_{s t d}}{v(t)}
$$

When the velocity is low or zero after performing the emergency braking, it could lead to really large $h_{d, \text { meas }}(t)$. To avoid this situation, a maximum allowed time gap $h_{d, \max }$ is set to saturate $h_{d, \text { meas }}(t)$.

The gap closing procedure consists of a time gap linear function depending on the inter-vehicle spacing $d(t)$ and the ego-speed $v(t)$. Then, two steps are followed to close the gap between the preceding and ego-vehicle:

- If the initial inter-vehicle distance $d_{r, s t a r t}$ is higher than the term $d_{r, \text { final }}=h_{d, \text { max }} v(t)$, a constant and comfortable acceleration $a_{g c}$ is applied until $d_{r, \text { final }}$ and the final velocity $v_{\text {final }}$ yield a current time gap equal to $h_{d, \max }\left(v_{\text {final }}\right.$ is limited as $\left.v_{\max }\right)$. The term $a_{g c}$ could be chosen depending on driver preferences: smooth, medium, hard; the higher $a_{g c}$ is set, the faster $h_{d, \max }$ will be reached. 


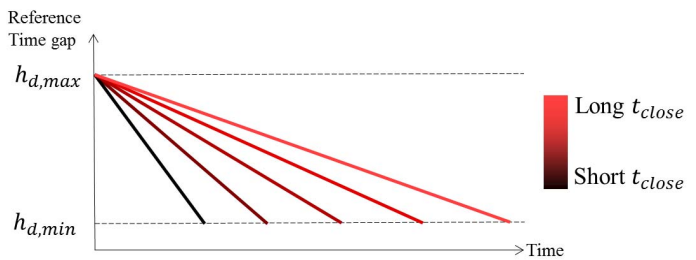

Fig. 8. Gap closing operation through the time gap adaptation.

TABLE I

Design PARAMETERS FOR THE GAP Closing MANEUVER

\begin{tabular}{|l|l||l|l|}
\hline Parameter & Value & Parameter & Value \\
\hline$K_{p}$ & 2.66 & $K_{d}$ & 0.79 \\
\hline$\alpha$ & 0.93 & $d_{\text {std }}$ & $5 \mathrm{~m}$ \\
\hline$h_{d, \max }$ & $5 s$ & $h_{d, \min }$ & $0.7 \mathrm{~s}$ \\
\hline$t_{\text {close }}$ & $15 \mathrm{~s}$ & $h_{d, A C C}$ & $1.35 \mathrm{~s}$ \\
\hline
\end{tabular}

- Once $h_{d, \max }$ is reached, ACC is executed having the desired time gap $h_{d}$ decreased until it reaches the ACC limit time gap $h_{d, A C C}$ and then the system switches to CACC to keep reducing up to the CACC desired value for car-following $\left(h_{d, \min }\right)$. The time gap decremental ratio depends directly on $t_{\text {close }}$, which is a design parameter that will define the gap closing time between the highest time gap $h_{d, \max }$ and $h_{d, \min }$. A suitable $t_{\text {close }}$ should be chosen considering the trade-off between passengers' comfort and time spent on the transition. The operation is shown in Fig. 8.

While the gap closing is carried out, it is important to guarantee that the communication link is resumed when $h_{d \text {,meas }}(t)=h_{d, A C C}$, where $h_{d, A C C}$ is set as the minimum time gap where system string stability is ensured for ACC.

Design parameters applied in the experimental results can be found in the table I.

\section{EXPERIMENTAL RESUlTS}

Three Cycabs and three pedestrians including one equipped with V2P communication are used for an experimental validation of the proposed system.

A speed profile emulating an urban driving scenario with several speed transitions is provided to the platoon leader, while the other two Cycabs are performing a carfollowing. The experiment is composed of several scenarios targeting the test and validation of environment detection, car-following, emergency braking and gap closing systems. Finally, a complete demonstration of all systems showing the integration and transitions is provided.

\section{A. Pedestrian Detection}

First tests are carried out to demonstrate platoon behavior when a pedestrian is walking towards the platoon trajectory.

Fig. 9 displays the distance to pedestrian tracked by V2P (blue line) and by LiDAR (red line) in the upper plot. It is shown that the V2P communication system can serve as a predictive tool since the the distance to pedestrian is available
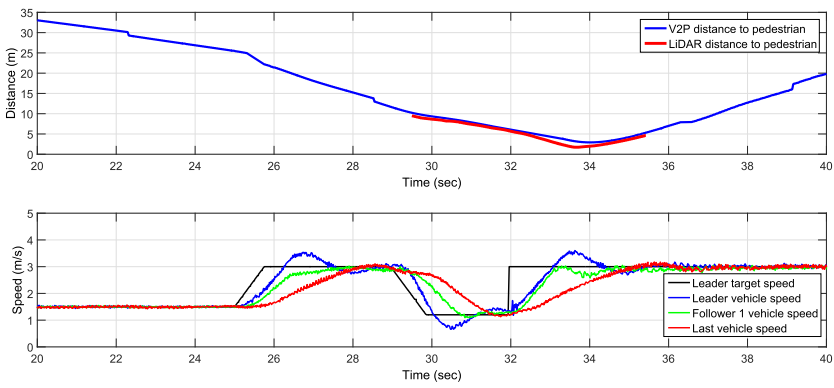

Fig. 9. Platoon performance when the leader vehicle detects a crossing pedestrian with the V2P system and the LiDAR, as well as the speed reduction zone (light blue background).

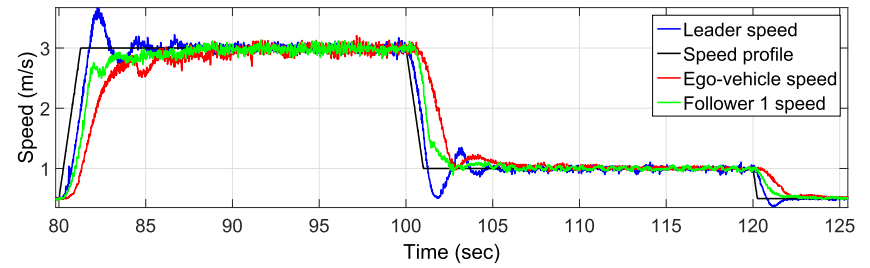

Fig. 10. Leader and followers performing car-following maneuvers in stop\&go scenarios.

even when this one is not visible for the LiDAR. Further information fusion between these two sources is then useful in urban environments where occlusion reduces the capability of LiDAR-based only protection systems.

Besides, as the first vehicle follows its reference speed profile, a speed reduction is performed correctly by the platoon leader upon the detection a possible collision (at time $\mathrm{t}=29 \mathrm{~s}$ ) as presented in the lower plot of Fig. 9. Once the pedestrian no longer represents an obstacle for the platoon (at time $t=32 \mathrm{~s}$ ), the desired speed profile is resumed.

\section{B. Car-Following}

As presented in Sec. IV-A.1, each follower vehicle implements a fractional-order controller and the time gap $h_{d, \min }=$ $0.7 \mathrm{sec}$ is applied so that string stability is ensured using a CACC car-following technique.

Fig. 10 shows the time response of the leader vehicle to the reference speed given by the speed profile and the rest of the string members regulating the inter-vehicle distances. The plot shows that the behavior results string stable, since during leader speed transitions the rest of the vehicles track their precedings' speed attenuating the oscillations. Such behavior would permit to extend the string size ensuring safety and comfort.

\section{Emergency Braking System}

Whenever a pedestrian crosses the platoon between the string members, the emergency braking state is activated. In this situation, the relative distance to the obstacle is output by the environment detection system and a stopping maneuver is started. Here, the distance to pedestrian, the reference and real speeds of the different vehicles are examined in Fig. 11 for a desired stopping distance $d_{\text {safety }}=1.5 \mathrm{~m}$. 


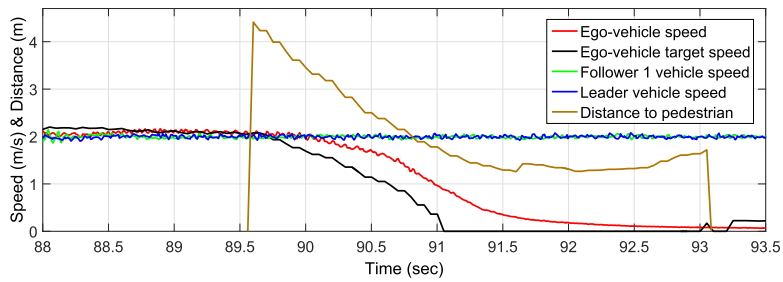

Fig. 11. Demonstration of the emergency braking maneuver with the control variables.
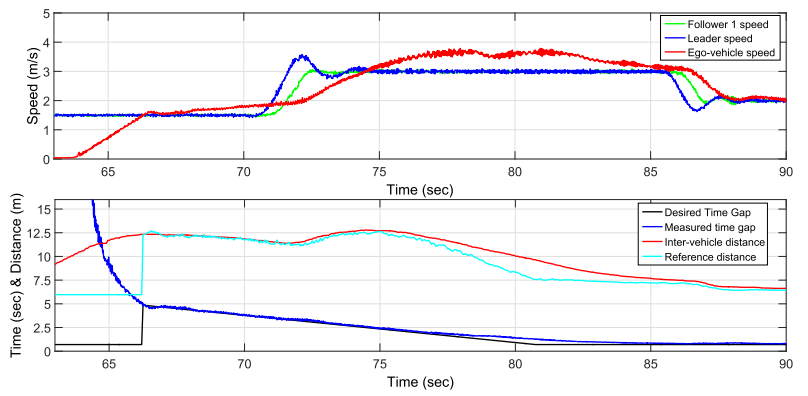

Fig. 12. a) Upper plot shows the performance of the three string members by showing each vehicles' speed. b) Lower plot depicts the control variables of the ego-vehicle.

The controller outputs the target speed (black line) to perform the maneuver, while the leader and first follower (blue and green lines) continue in car-following state. The brown line shows how the ego-vehicle is quickly approaching the pedestrian, but the displacement rate decreases during the braking until it stops at $d_{\text {safety }}$ from the obstacle. Finally, the vehicle stands still waiting for the pedestrian to go out of the platoon corridor and switch to the gap closing state.

\section{Gap-Closing}

After the pedestrian is out of the platoon corridor, the egovehicle has to close the gap with respect to the preceding car through the time gap manipulation. In Fig. 12 two plots are presented, the upper one shows the general platoon overview with the string members' speeds. The lower plot details control variables such reference and current spacing, and desired and measured time gap.

Firstly, the vehicle starts the platoon rejoining maneuver by accelerating with an $a_{g c}=1.5 \mathrm{~m} / \mathrm{s}^{2}$ (time $t=62 \mathrm{sec}$ to $\mathrm{t}=67 \mathrm{sec}$ ), arriving to the gap-closing time gap range (between $h_{d, \max }$ and $h_{d, \min }$ ). Then, the time gap (blue line) decreases as the speed increases until the target identification process is completed and the reference time gap reduction starts from $h_{d, \max }$ to $h_{d, \min }$ with a $t_{c l o s e}=15 \mathrm{sec}$. This process is depicted by the reference time gap $h_{d}$ (black line) and the real time gap $h_{d, \text { meas }}$. In the upper plot, one can highlight how the gap closing maneuver requires the vehicle to speed up at the beginning and go faster than the other string members to rejoin and reduce the inter-distance, even if the leader vehicle also accelerates (time $\mathrm{t}=72 \mathrm{sec}$ ). The platoon coupling is carried out in a stable and smooth way as can be noticed from time $\mathrm{t}=80 \mathrm{sec}$ to $\mathrm{t}=90 \mathrm{sec}$; when finally the ego-vehicle is again at the car-following state with the desired time gap $h_{d, \min }$.
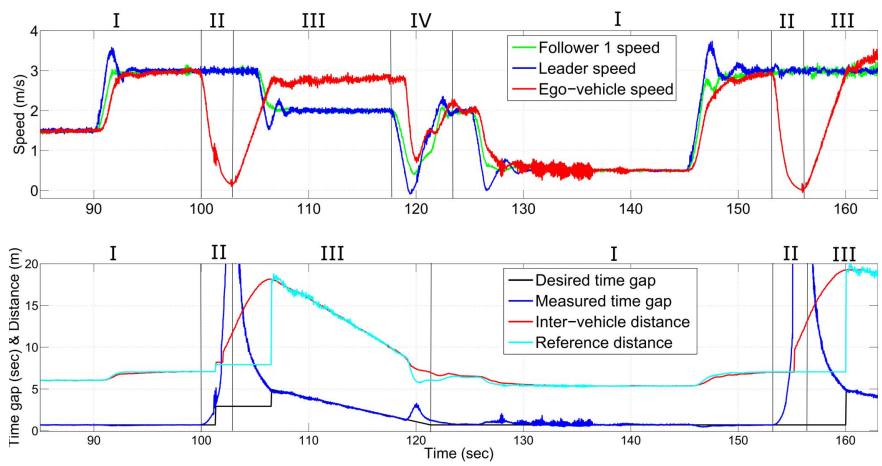

Fig. 13. State machine integration: V2P protection maneuver (IV), car-following (I), emergency braking (II) and gap-closing (III). Their transitions are shown through a speed plot (upper plot) and the control variables of the ego-vehicle (lower plot).

\section{E. State Machine Demonstration}

A complete scenario has been set to evaluate the transitions between the three states that composes the state machine. Fig. 13 shows the three vehicles string performing a CACC car-following with a time gap of $h_{d, \min }=0.7 \mathrm{sec}$ as desired. Suddenly the last follower vehicle performs an emergency stopping due to a pedestrian that crossed in front (time $\mathrm{t}=100 \mathrm{sec}$ ), and the speed decreases. Time gap with respect to the preceding vehicle is reduced when performing the gap-closing state (time $t=103 \mathrm{sec}$ to $t=121 \mathrm{sec}$ ). During this period, another pedestrian crosses but this time in front of the leader vehicle; which requires the employment of the pedestrian detection system to stop following the speed profile and reduce the platoon speed to avoid further collision with it. From time $\mathrm{t}=121 \mathrm{sec}$ to $\mathrm{t}=154 \mathrm{sec}$, the car-following state is correctly resumed by following the speed profile in a string stable way. Afterwards, another pedestrian goes through the platoon corridor (time 154-157 sec) which activates the emergency braking system followed by the gap-closing when the pedestrian exits the ego-corridor.

\section{Conclusions}

A modular system was presented for a safe platoon in urban environments while considering interaction with pedestrians. Here, V2P provided a predictive tool to detect collision with pedestrians, which is crucial in urban cluttered environment where LiDARs have limited visibility. It allowed the leader vehicle to avoid upcoming collisions by slowing the speed rather than abruptly braking when pedestrians were visible.

A robust fractional-order controller was developed to handle car-following maneuvers with short inter-vehicle time gaps, ensuring the string stability. Furthermore, a state machine is designed to deal with platoon transitions from car-following CACC to string decoupling due to an emergency stop maneuver in case of pedestrian crossing inside the platoon corridor. The mentioned state machine is set to perform a string rejoining when the pedestrian is out of the corridor. This is managed through a gap-closing maneuver that implies firstly to accelerate until the preceding vehicle is at a maximum range and perform a time gap reduction to the desired value, allowing 
to resume the car-following state. Encouraging tests in real platforms demonstrate the correct integration of the described perception and control systems. The experiments also show the performance of the leader vehicle pedestrian detection system as well as the correct behavior of the implemented machine states: car-following, emergency braking and gapclosing; inside the follower vehicles.

\section{ACKNOWLEDGMENTS}

The authors express their gratitude to the RITS Team for the support of this work.

\section{REFERENCES}

[1] S.-H. Sun, J.-L. Hu, Y. Peng, X.-M. Pan, L. Zhao, and J.-Y. Fang, "Support for vehicle-to-everything services based on LTE," IEEE Wireless Commun., vol. 23, no. 3, pp. 4-8, Jun. 2016.

[2] V. Milanés, S. Shladover, J. Spring, C. Nowakowski, H. Kawazoe, and M. Nakamura, "Cooperative adaptive cruise control in real traffic situations," IEEE Trans. Intell. Transp. Syst., vol. 15, no. 1, pp. 296-305, Feb. 2014.

[3] J. Godoy, V. Milanés, J. Pérez, J. Villagrá, and E. Onieva, "An auxiliary V2I network for road transport and dynamic environments," Transp. Res. C, Emerg. Technol., vol. 37, pp. 145-156, Dec. 2013.

[4] V. Milanés and S. Shladover, "Modeling cooperative and autonomous adaptive cruise control dynamic responses using experimental data," Transp. Res. C, Emerg. Technol., vol. 48, pp. 285-300, Nov. 2014.

[5] M. Chan, "Global status report on road safety," World Health Org., Geneva, Switzerland, Tech. Rep., 2015.

[6] V. Milanés et al., "Vision-based active safety system for automatic stopping," Expert Syst. Appl., vol. 39, no. 12, p. $11234-11242,2012$.

[7] X. Song, J. Cui, H. Zhao, H. Zha, and R. Shibasaki, "Laser-based tracking of multiple interacting pedestrians via on-line learning," Neurocomputing, vol. 115, pp. 92-105, Sep. 2013.

[8] V. Milanés, L. Alonso, J. Villagrá, J. Godoy, T. de Pedro, and J. P. Oria, "Traffic jam driving with NMV avoidance," Mech. Syst. Signal Process., vol. 31, pp. 332-344, Aug. 2012.

[9] A. Broggi, P. Cerri, S. Ghidoni, P. Grisleri, and H. G. Jung, "A new approach to urban pedestrian detection for automatic braking," IEEE Trans. Intell. Transp. Syst., vol. 10, no. 4, pp. 594-605, Dec. 2009.

[10] H. Hamdane, T. Serre, C. Masson, and R. Anderson, "Issues and challenges for pedestrian active safety systems based on real world accidents," Accident Anal. Prevention, vol. 82, pp. 53-60, Sep. 2015.

[11] A. Petrovskaya and S. Thrun, "Model based vehicle detection and tracking for autonomous urban driving," Auton. Robots, vol. 26, nos. 2-3, pp. 123-139, Apr. 2009.

[12] F. Güney and A. Geiger, "Displets: Resolving stereo ambiguities using object knowledge," in Proc. IEEE Conf. Comput. Vis. Pattern Recognit. (CVPR), Jun. 2015, pp. 4165-4175.

[13] J. J. Anaya, P. Merdrignac, O. Shagdar, F. Nashashibi, and J. E. Naranjo, "Vehicle to pedestrian communications for protection of vulnerable road users," in Proc. IEEE Intell. Vehicles Symp. (IV), Jun. 2014, pp. 1037-1042.

[14] L. Zhenyu, P. Lin, Z. Konglin, and Z. Lin, "Design and evaluation of V2X communication system for vehicle and pedestrian safety," J. China Univ. Posts Telecommun., vol. 22, no. 6, pp. 18-26, 2015.

[15] J. J. Martinez and C. Canudas-de-Wit, "A safe longitudinal control for adaptive cruise control and stop-and-go scenarios," IEEE Trans. Control Syst. Technol., vol. 15, no. 2, pp. 246-258, Mar. 2007.

[16] V. Milanes, J. Villagra, J. Godoy, and C. Gonzalez, "Comparing fuzzy and intelligent PI controllers in stop-and-go manoeuvres," IEEE Trans. Control Syst. Technol., vol. 20, no. 3, pp. 770-778, May 2012.

[17] I. Moon, S. Moo, and K. Yi, "Design, tuning, and evaluation of a fullrange adaptive cruise control system with collision avoidance," Control Eng. Pract., vol. 17, pp. 442-455, Apr. 2009.

[18] P. Resende, E. Pollard, H. Li, and F. Nashashibi, "Low speed automation: Technical feasibility of the driving sharing in urban areas," in Proc. IEEE Int. Conf. Intell. Transp. Syst. (ITSC), Oct. 2013, pp. 442-448.

[19] U. Ramer, "An iterative procedure for the polygonal approximation of plane curves," Comput. Graph. Image Process., vol. 1, no. 3, pp. 244-256, Nov. 1972 .

[20] J. Hershberger and J. Snoeyink, "An O(nlogn) implementation of the Douglas-Peucker algorithm for line simplification," in Proc. ACM Anпu. Symp. Comput. Geometry, 1994, pp. 383-384.
[21] V. Nguyen, A. Martinelli, N. Tomatis, and R. Siegwart, "A comparison of line extraction algorithms using 2D laser rangefinder for indoor mobile robotics," in Proc. IEEE/RSJ Int. Conf. Intell. Robots Syst. (IROS), Aug. 2005, pp. 1929-1934.

[22] R. Domínguez, E. Onieva, J. Alonso, J. Villagra, and C. González, "LIDAR based perception solution for autonomous vehicles," in Proc. Int. Conf. Intell. Syst. Design Appl. (ISDA), 2011, pp. 790-795.

[23] P. Merdrignac, E. Pollard, and F. Nashashibi, "2D laser based road obstacle classification for road safety improvement," in Proc. IEEE Int. Workshop Adv. Robot. Soc. Impacts (ARSO), Jun. 2015, pp. 1-6.

[24] M. Bagheri, M. Siekkinen, and J. K. Nurminen, "Cellular-based vehicle to pedestrian (V2P) adaptive communication for collision avoidance," in Proc. IEEE Int. Conf. Connected Vehicles Expo (ICCVE), Nov. 2014, pp. $450-456$.

[25] K. David and A. Flach, "Car-2-X and pedestrian safety," IEEE Veh. Technol. Mag., vol. 5, no. 1, pp. 70-76, Mar. 2010.

[26] P. Merdrignac, O. Shagdar, I. B. Jemaa, and F. Nashashibi, "Study on perception and communication systems for safety of vulnerable road users," in Proc. IEEE Int. Conf. Intell. Transp. Syst. (ITSC), Sep. 2015, pp. $1876-1881$.

[27] Intelligent Transport Systems (ITS); Vehicular Communications; Basic Set of Applications; Part 2: Specification of Cooperative Awareness Basic Service, document, Transport ETSI, 2011.

[28] E. Kaplan and C. Hegarty, Understanding GPS: Principles and Applications. Norwood, MA, USA: Artech House, 2005.

[29] M. Liebner, F. Klanner, and C. Stiller, "Active safety for vulnerable road users based on smartphone position data," in Proc. IEEE Intell. Vehicles Symp. (IV), 2013, pp. 256-261.

[30] A. Yilmaz, O. Javed, and M. Shah, "Object tracking: A survey," ACM Comput. Surv., vol. 38, no. 4, Dec. 2006, Art. no. 13.

[31] Y. Wu, J. Lim, and M.-H. Yang, "Online object tracking: A benchmark," in Proc. IEEE Conf. Comput. Vis. Pattern Recognit. (CVPR), Jun. 2013, pp. 2411-2418.

[32] M. J. Quinlan and R. H. Middleton, "Multiple model Kalman filters: A localization technique for RoboCup soccer," in Robot Soccer World Cup XIII. Berlin, Germany: Springer, 2010, pp. 276-287.

[33] P. C. Mahalanobis, "On the generalized distance in statistics," Proc. Nat. Inst. Sci. India, vol. 2, no. 1, pp. 49-55, 1936.

[34] D. Calzolari, B. Schürmann, and M. Althoff, "Comparison of trajectory tracking controllers for autonomous vehicles," in Proc. IEEE 20th Int. Conf. Intell. Transp. Syst. (ITSC), Oct. 2017, pp. 1-8.

[35] D. Swaroop and K. R. Rajagopal, "A review of constant time headway policy for automatic vehicle following," in Proc. IEEE Int. Conf. Intell. Transp. Syst. (ITSC), Aug. 2001, pp. 65-69.

[36] R. Kianfar, P. Falcone, and J. Fredriksson, "A receding horizon approach to string stable cooperative adaptive cruise control," in Proc. IEEE Int. Conf. Intell. Transp. Syst. (ITSC), Oct. 2011, pp. 734-739.

[37] X.-Y. Lu, J. K. Hedrick, and M. Drew, "ACC/CACC-control design, stability and robust performance," in Proc. IEEE Amer. Control Conf. (ACC), vol. 6, May 2002, pp. 4327-4332.

[38] J. Ploeg, D. P. Shukla, N. van de Wouw, and H. Nijmeijer, "Controller synthesis for string stability of vehicle platoons," IEEE Trans. Intell. Transp. Syst., vol. 15, no. 2, pp. 854-865, Apr. 2014.

[39] Y. Liu, B. Xu, and Y. Ding, "Convergence analysis of cooperative braking control for interconnected vehicle systems," IEEE Trans. Intell. Transp. Syst., vol. 18, no. 7, pp. 1894-1906, Jul. 2017.

[40] J. Ploeg, E. Semsar-Kazerooni, G. Lijster, N. van de Wouw, and H. Nijmeijer, "Graceful degradation of cooperative adaptive cruise control," IEEE Trans. Intell. Transp. Syst., vol. 16, no. 1, pp. 488-497, Feb. 2015.

[41] G. J. L. Naus, R. P. A. Vugts, J. Ploeg, M. J. G. van de Molengraft, and M. Steinbuch, "String-stable CACC design and experimental validation: A frequency-domain approach," IEEE Trans. Veh. Technol., vol. 59, no. 9, pp. 4268-4279, Nov. 2010.

[42] H. Li, Y. Luo, and Y. Chen, "A fractional order proportional and derivative (FOPD) motion controller: Tuning rule and experiments," IEEE Trans. Control Syst. Technol., vol. 18, no. 2, pp. 516-520, Mar. 2010.

[43] C. Flores, V. Milanés, and F. Nashashibi, "Using fractional calculus for cooperative car-following control," in Proc. IEEE Int. Conf. Intell. Transp. Syst. (ITSC), Nov. 2016, pp. 907-912.

[44] D. F. Llorca et al., "Autonomous pedestrian collision avoidance using a fuzzy steering controller,' IEEE Trans. Intell. Transp. Syst., vol. 12, no. 2, pp. 390-401, Jun. 2011.

[45] F. Bu, H.-S. Tan, and J. Huang, "Design and field testing of a cooperative adaptive cruise control system," in Proc. IEEE Amer. Control Conf. (ACC), Jun. 2010, pp. 4616-4621. 


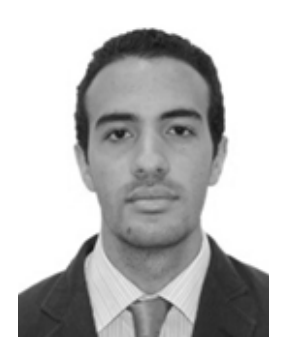

Carlos Flores was born in Caracas, Venezuela, in 1992. He received the B.Sc. degree in electronics engineering from Simón Bolívar University, Venezuela, in 2014. He is currently pursuing the Ph.D. degree with the RITS Team, inria, Paris, France, and Mines ParisTech, PSL Research University, France.

His research interests include automated vehicles, cooperative driving, system modeling and control, and fractional-order calculus.

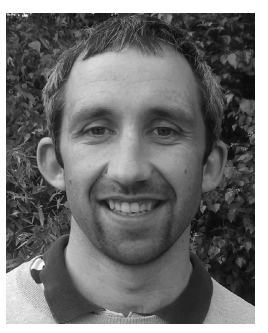

Pierre Merdrignac (M'16) received the M.E degree from Telecom Bretagne, Brest, in 2012, and the Ph.D. degree in robotics from Mines ParisTech, the RITS Team, inria, Paris, and the VEDECOM Institute, in 2015. He was a Research Engineer with inria from 2015 to 2016. Since 2016, he has been a Researcher with the VEDECOM Institute. His research interests include vehicular communication, environment perception, and multi-source information fusion.

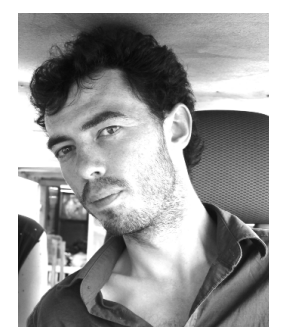

Raoul de Charette received the M.Sc. degree in computer graphics and art from Paris 8 University and the Ph.D. degree in computer vision for autonomous vehicles from Mines ParisTech, Paris, France, in 2012. He was consecutively with Mines ParisTech, Carnegie Mellon University, Pittsburgh, USA, and the University of Makedonia, Thessaloniki, Greece. He is currently leading the Computer Vision Group, RITS Team, inria. His researches focus on scene understanding, vision in degraded weather, end-to-end perception, vision for autonomous driving, object reconstruction, and so on. He received the Best Paper Award from ICCP 2012.

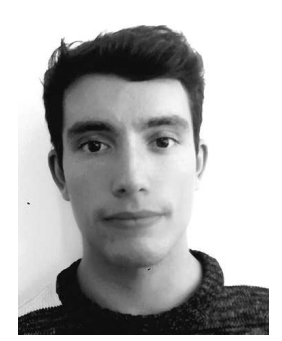

Francisco Navas was born in Badajoz, Spain, in 1992. He received the B.Sc. degree in electrical engineering from the University of Extremadura, Spain, in 2014, and the M.Sc. degree in advance electronics systems and intelligent systems from the University of Alcalá, Madrid, Spain, in 2015.

$\mathrm{He}$ is currently pursuing the Ph.D. degree with the RITS Team, inria, Paris, and Mines ParisTech, PSL Research University. His research interests include autonomous vehicles, plug\&play control, switching controllers, modeling, and vehicle-infrastructure cooperation.

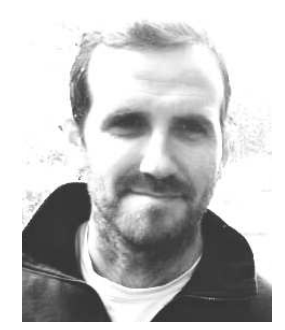

Vicente Milanés received the Ph.D. degree in electronic engineering from the University of Alcalá, Madrid, Spain, in 2010. He was with the AUTOPIA program, Center for Automation and Robotics, UPM-CSIC, Spain, from 2006 to 2011. In 2014, he joined the RITS Team, inria, France. Since 2016, he has been with the Research Department, Renault SAS, France. His research interests include autonomous vehicles, vehicle dynamic control, intelligent traffic and transport infrastructures, and vehicle-infrastructure cooperation. He was a recipient of a two-year Fulbright Fellowship at the California PATH, UC Berkeley. He received the best paper award in three conferences and three major awards for his Ph.D. degree.

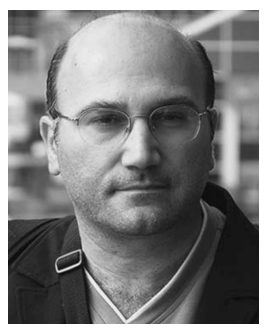

Fawzi Nashashibi received the master's degree in automation, industrial engineering, and signal processing from the Laboratory for Analysis and Architecture of Systems/Centre Nationnal de la Recherche Scientifique (LAAS/CNRS), Toulouse, France, the Ph.D. degree in robotics from the LAAS/CNRS Laboratory, Toulouse University, and the HDR Diploma (accreditation to research supervision) from Pierre-and-Marie-Curie University (Paris 6), Paris, France. Since 1994, he has been a Senior Researcher and the Program Manager with the Robotics Center, Mines ParisTech, Paris. He has also been a Lecturer with several universities (Mines ParisTech, Paris 8 Saint-Denis, Leonard de Vinci University ESILV professor, Telecom Sud Paris, INT Evry, Ecole Centrale de Electronique) in the fields of image and signal processing, 3-D perception, 3 -D infographics, mobile robotics, and $\mathrm{C}++/ \mathrm{JAVA}$ programming. Since 2000, he has been a Research and Development Engineer and a Project Manager with ARMINES. Since 2010, he has been a Senior Researcher and a Program Manager with the RITS Team, inria, Paris-Rocquencourt, France. He was a Research Engineer with PROMIP, involved in mobile robotics perception dedicated to space exploration, and a Technical Manager with Light Company, where he led the developments of virtual reality/augmented reality applications. He played key roles in over 50 European and national French projects, such as Carsense, HAVE-it, PICAV, CityMobil, ARCOS, $\mathrm{ABV}$, LOVe, and SPEEDCAM, some of which he has coordinated. He is also involved in many collaborations with French and international academics and industrial partners. He is the author of numerous publications and patents in the field of ITS and ADAS systems. His current research focuses on advanced urban mobility through the design and development of highly automated transportation systems, which includes highly automated unmanned guided vehicles (such as cybercars) as well automated personal vehicles. In this field, he is known as an international expert. His main research interests include environment perception and multisensor fusion, vehicle positioning, and environment 3-D modeling with main applications in intelligent transport systems and robotics. He is a member of the IEEE ITS Society and the Robotics and Automation Society. He is an Associate Editor of several IEEE international conferences, such as ICRA, IROS, IV, ITSC, and ICARCV. 